\title{
Les Cyanophyceae marines de l'île de Ngor (Sénégal)
}

\author{
Madiop GUEYE*, Ngansoumana BA, Moussa Yagame BODIAN, Mame Samba MBAYE, \\ César BASSENE, Maïmouna KANE, Kandioura NOBA et Ablaye NGOM
}

\author{
Laboratoire de Botanique et Biodiversité, Département de Biologie Végétale, Faculté des Sciences et \\ Techniques, Université Cheikh Anta DIOP, BP 5005, Dakar-Fann, Sénégal. \\ *Auteur correspondant, E-mail : madiopguey@yahoo.fr ; Tel : (221) 776121914
}

\section{RÉSUMÉ}

La présente étude a été initiée dans le but de déterminer la composition taxonomique et la structure des peuplements de Cyanophyceae au niveau de l'ile de Ngor. Durant les travaux, une description basée sur des caractères morphologiques du thalle (cellule, colonie ou filament) des taxa a été effectuée. Aussi une classification et un inventaire systématique de ces taxa ont été réalisés. Six prélèvements ont été effectués entre juillet 2009 et août 2010 dans les eaux marines de l'île de Ngor située à $800 \mathrm{~m}$ de la côte dakaroise (Sénégal). L'observation des différents échantillons prélevés a permis de répertorier au niveau de cet écosystème 38 taxa de Cyanophyceae dont 28 identifiées jusqu'au niveau spécifique, réparties en 18 genres et 11 familles. Sur ces 11 familles, seules trois d'entre elles regroupent plus de la moitié des taxa présents. Ce sont les Oscillatoriaceae avec 31,56\%, les Phormidiaceae et les Pseudanabaenaceae avec chacune 15,79\%. Les Merismopediaceae représentent 10,53\% des taxa, les Chroococcaceae 7,89\%, et les Synechococcaceae 5,26\%. Les familles des Borziaceae, des Xenococcaceae, des Hyellaceae, des Microcystaceae et des Rivulariaceae ne représentent chacune que 2,63\%. Pour ce qui est des genres, Oscillatoria Vaucher est le plus représenté avec 7 taxa, suivi des genres Lyngbya C. Agardh et Phormidium Kütz. Ex Gom. avec chacun 4 taxa. Sur les 28 taxa identifiés jusqu'au niveau espèce, 17 ont été décrits pour la $1^{\text {ere }}$ fois au Sénégal.

(C) 2014 International Formulae Group. All rights reserved.

Mots-clés : Cyanophyceae, composition taxonomique, structure, île de Ngor, Sénégal.

\section{INTRODUCTION}

Pour suivre les recommandations de la Conférence des Nations Unies sur l'Environnement et le Développement (CNUED), tenue à Rio en juin 1992 et dont l'un des objectifs était la conservation de la diversité biologique, le Sénégal, à l'instar de plusieurs pays africains, s'est investi dans la réalisation d'une monographie.

Les résultats obtenus ont montré que dans les écosystèmes terrestres, le Sénégal présente une diversité spécifique importante avec une flore phanérogamique constituée par environ 2500 espèces (Ba et Noba, 2001). Cependant, il existe plusieurs groupes végétaux (aquatiques et terrestres) présents au Sénégal qui n'ont pas été étudiés (bryophytes) ou qui ont fait l'objet de rares études (Cyanophyta et algues).

Pour ce qui est des macroalgues, les quelques études systématiques menées ont été réalisées en milieu marin par Dangeard 
(1952), Bodard et Mollion (1974), Harper et Garbary (1997), Bodian (2000) et Diedhiou (2010).

Quant aux Cyanophyta et microalgues, les études réalisées ont porté essentiellement sur les milieux d'eau douce. Ce sont les travaux de Guermeur (1954), de De Poucques (1956), de Dia et Reynaud (1982), de Compère (1991), de Cogels et Gac (1995), de Carlbro (1999), de Berger et al. (2005), de Ba et al. (2006) et de Gueye et al. (2013).

$\mathrm{Au}$ Sénégal, malgré l'importance du réseau hydrographique et de la diversité des écosystèmes marins rencontrés au niveau des côtes $(700 \mathrm{~km})$, des estuaires, les Cyanophyta et microalgues y sont très peu étudiés. Excepté les travaux de Leuduger-Formorel (1898) et d'Amossé (1970) sur les diatomées et ceux de Dia (1983) sur la biologie et biomasse du phytoplancton, ces microorganismes n'ont pas fait l'objet d'une autre étude au Sénégal.

Il apparaît alors nécessaire de renforcer les connaissances sur ces microorganismes végétaux qui suscitent une attention particulière à travers le monde du fait non seulement de leurs rôles dans les écosystèmes marins (producteurs primaires) mais de leur exploitation dans les domaines de production énergétique (bioproduction d'hydrogène, biocarburant) et de la nuisance de certaines espèces qui secrètent des toxines (Dinophyceae et de Cyanophyceae).

C'est dans ce contexte que s'inscrit la présente étude dont l'objectif principal est de déterminer la composition taxonomique et la structure des peuplements de Cyanophyceae au niveau de l'île de Ngor.

\section{MATERIEL ET METHODES}

\section{Présentation de l'île de Ngor}

C'est une petite île qui se trouve à 800 $\mathrm{m}$ de la côte dakaroise à $14^{\circ} 45^{\prime} 30^{\prime}$ ' $\mathrm{N}$ et $17^{\circ} 30^{\prime} 56^{\prime}$ 'W (Figure 1). Elle est située à l'Ouest du village de Ngor au milieu d'une baie protégée. L'île est constituée par les coulées d'hawaïte à texture doléritique du volcanisme basaltique quaternaire des Mamelles.

Elle appartient à la zone micro climatique de la grande côte sénégalaise qui, au sein du domaine intertropical présente des particularités qui la différencient de l'intérieur du pays. Cette zone est soumise aux effets de masses d'airs (alizé, mousson, harmattan) déterminées par les champs de pressions (les anticyclones des Açores, de Sainte-Hélène et de Libye et de la dépression saharienne).

Elle bénéficie, du fait de sa situation côtière, de températures idéales $\left(25{ }^{\circ} \mathrm{C}\right)$ pendant presque toute l'année avec des pointes de $18{ }^{\circ} \mathrm{C}$ mini en février et de $32{ }^{\circ} \mathrm{C}$ maxi en Octobre.

\section{Prélèvement des échantillons}

Les échantillons de Cyanophyta ont été prélevés sur la plage de l'île de Ngor et de celle de Ngor-village au niveau des sites NG1 (1445'03.9', $\mathrm{N} ; \quad$ 017 30'54.0', W), NG2 $\left(14^{\circ} 45^{\prime} 18.4^{\prime \prime} \mathrm{N} ; 017^{\circ} 30^{\prime} 45.1^{\prime \prime} \mathrm{W}\right)$ et NG3 $\left(14^{\circ} 45^{\prime} 12.1^{\prime \prime} \mathrm{N} ; \quad 017^{\circ} 30^{\prime} 45.4^{\prime}, \mathrm{W}\right)$. Les échantillonnages ont été faits entre juillet 2009 et août 2010 avec 6 sorties effectuées à intervalle irrégulier (18 juillet 2009, 9 janvier 2010, 14 mars 2010, 5 mai 2010, 9 juin 2010, 9 juillet 2010, 28 août 2010).

Les récoltes ont été réalisées à l'aide d'un filet à plancton de $10 \mu \mathrm{m}$ de maille à la surface des plans d'eau par trainée horizontale pour les espèces planctoniques et par grattage de la surface de tout support immergé présent au niveau de la station pour les espèces fixées. Une partie de l'échantillon prélevé a été recueillie dans des flacons de $60 \mathrm{ml}$ puis immédiatement fixés avec du formol à $4 \%$.

\section{Description et identification des specimens Description des spécimens}

Après le dépôt au fond du flacon des individus, une goutte d'eau est prélevée puis 
observée au microscope (Motic Digital Microscope) à l'objectif d'immersion (X100). Des dessins ont été réalisés ainsi que des mesures de dimensions grâce à un oculaire (X10) micrométrique étalonné par une lame micrométrique.

\section{Identification des spécimens}

A partir des dessins faits à main levée, de la description basée sur les caractères morphologiques et des mesures de dimensions, une identification des taxa a été faite en comparant nos données à certains travaux comme ceux de Naskar et al. (2008), Crispino et Sant'Anna (2006), Nogueira et al. (2001), Da Rodda et Parodi (2005), BerardTherriault et al. (1999), Silva et al. (1996), Abbott et al. (1992), D'hont et Coppejan (1988) et Park et Dixon (1964).

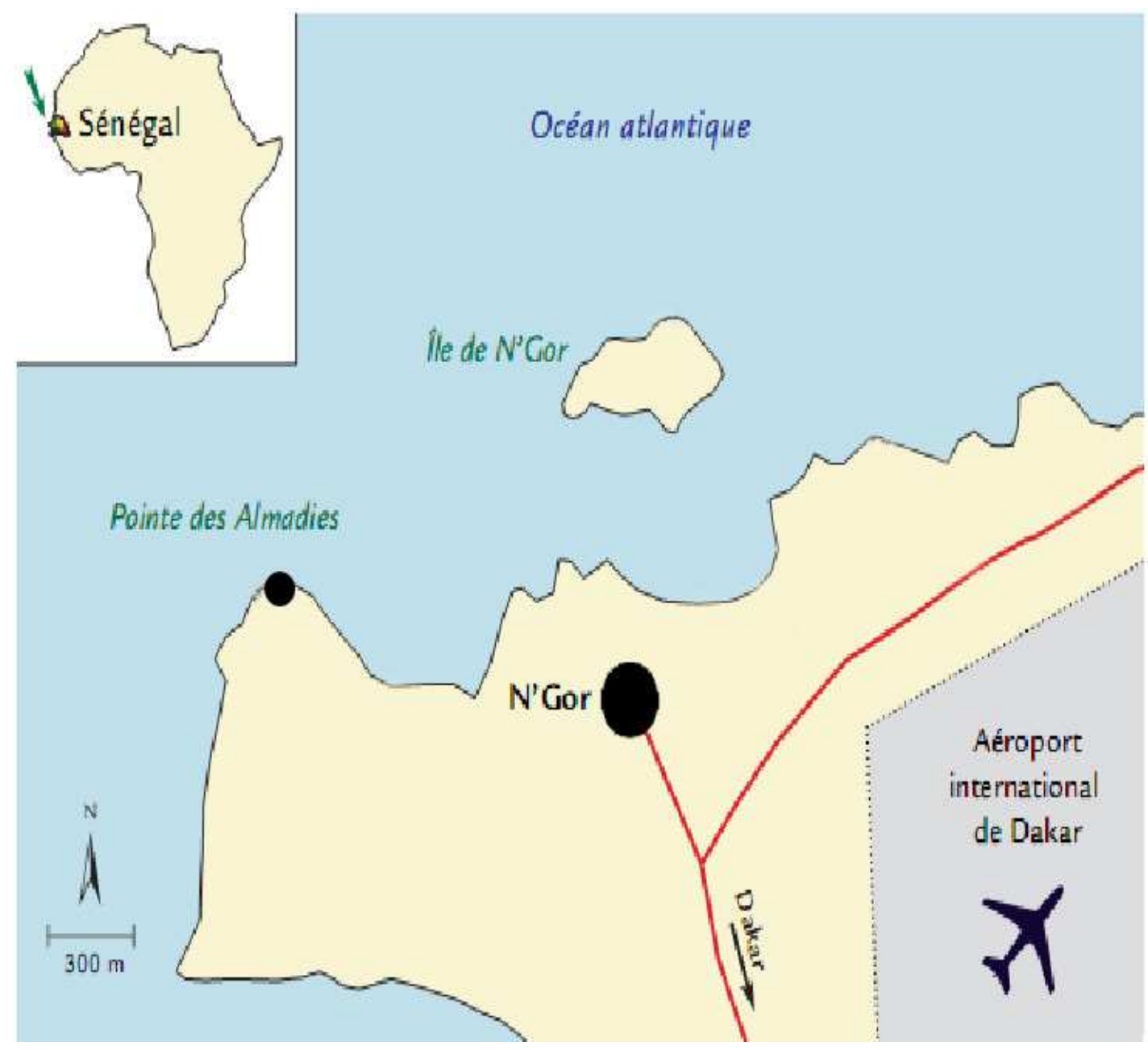

Figure 1 : Localisation de l'île de Ngor. 


\section{RESULTATS}

L'observation des différents échantillons a permis d'identifier au niveau de l'île de Ngor 38 taxa de Cyanophyceae répartis en 18 genres et 11 familles suivant la classification de Komárek et Hauer (2011) basée sur des caractères morphologiques.

En termes de nombre de taxa, la famille des Oscillatoriaceae est la plus importante avec $31,56 \%$; elle est suivie de celles des Phormidiaceae et des Pseudanabaenaceae avec chacune $15,79 \%$ des taxa recensées. Les familles des des Merismopediaceae forment $10,53 \%$ de l'ensemble des taxa. Le genre Oscillatoria Vaucher est le plus représenté avec 7 taxa dont 6 identifiés jusqu'au niveau spécifique, suivi des genres Lyngbya C. Agardh et Phormidium Kütz. Ex Gom avec chacun 4 taxa.

\section{Famille des Chroococcaceae}

Genre Chroococcus Nägeli

Chroococcus minor (Kützing) Nägeli (Figure 2)

\section{Syn. Protococcus minor Kützing}

L'espèce est formée de 3 à 4 petites cellules hémisphériques à subsphériques avec de très fines granulations. Leur diamètre est de 4-4,5 $\mu \mathrm{m}$. Le diamètre de la gelée est de 9 $\mu \mathrm{m}$. La description correspond à celle donnée par John et al. (2002) et D'hont et Coppejans (1988).

Chroococcus minutus (Kützing) Nägeli (Figure 3)

Quatre cellules subsphériques regroupées par deux et pourvues de granulations forment l'espèce; elles ont une couleur vert-jaune, tendant vers le beige. Leur diamètre est de $8 \mu \mathrm{m}$ et leur longueur varie entre 9 et $10 \mu \mathrm{m}$. Ces cellules observées présentent le même diamètre que celles décrites par John et al. (2002).

Chroococcus varius A. Braun (Figure 4)

Cellules de couleur verte regroupées par 4 en une petite colonie et pourvues d'une sorte de membrane individuelle lamellaire. Le diamètre des cellules sphériques est de $3 \mu \mathrm{m}$,

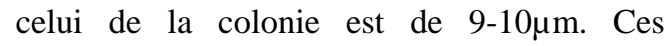
caractères sont également notés par Naz et al. (2004) et John et al. (2002).

\section{Famille des Xenococcaceae}

Genre Myxosarcina Printz

Myxosarcina sp (Figure 5)

Thalle de couleur bleu noirâtre, formé de plusieurs cellules pas totalement sphériques (parfois polygonales) parfois regroupées par 4 (en division) ou plus en sorte de glomérules. Le diamètre du thalle est environ égal à 27 $\mu \mathrm{m}$, celui des cellules varie entre 2 et $3 \mu \mathrm{m}$.

\section{Famille des Hyellaceae}

Genre Pleurocapsa Thuret

Pleurocapsa sp (Figure 6)

Espèce de couleur verte formée de plusieurs cellules sphériques, parfois polygonales regroupées en un amas irrégulier. Leur diamètre varie entre 3 et $7 \mu \mathrm{m}$.

\section{Famille des Merismopediaceae}

Genre Aphanocapsa Nägeli

Aphanocapsa littoralis Hansgirg (Figure 7)

Le thalle est formé de 7 à 8 cellules sphériques groupées en colonie, de manière compacte; les cellules présentent des granulations. Leur diamètre est de 6-7 $\mu \mathrm{m}$. La description correspond à celle donnée par D'hont et Coppejans (1988).

Aphanocapsa marina Hansgirg (Figure 8)

Syn.: Microcystis marina (Hansgirg) Kosinnskaja

Petite colonie amorphe formée de cellules sphériques d'environ $0,5 \mu \mathrm{m}$ de diamètre et entourée d'un fin mucilage. Les cellules sont pourvues de granulations et densément groupées. Les cellules ont le même diamètre que celles de l'espèce décrite par John et al. (2002).

Genre Merismopedia Meyen

Merismopedia minima G. Beck (Figure 9)

L'espèce se présente sous forme de petite colonie plate tabulaire, avec 4 cellules subsphériques. Leur diamètre est d'environ 1 $\mu \mathrm{m}$ et est légèrement inférieur au diamètre des cellules de l'espèce décrite par $\mathrm{Naz}$ et al. (2004).

Merismopedia mediterranea Nägeli (Figure 10)

Syn. : Merismopedia glauca $f$. mediterranea (Nägeli) Collins

Les cellules sont sphériques à elliptiques formant une colonie plate, 
tabulaire. Elles sont disposées très régulièrement en rangées verticales et horizontales. Leur diamètre est de 5-6 $\mu \mathrm{m}$. Merismopedia mediterranea présente les mêmes caractéristiques que les types décrits par Crispino et Sant'Anna (2006) et D'hont et Coppejans (1988).

\section{Famille des Synechococcaceae}

Genre Rhabdoderma Schmidle et Lauterborn

Rhabdoderma lineare Schmidle et Lauterborn (Figure 11)

Syn. : Gloeothece linearis Nägeli

Les cellules regroupées en colonie sont cylindriques à bords arrondies, parfois légèrement courbées. Elles sont légèrement colorées avec des granulations noires à grises. Leur longueur est de 11-12 $\mu \mathrm{m}$, leur diamètre de 3 à $4 \mu \mathrm{m}$. Notre échantillon a le même ordre de grandeur que celui de Sant'Anna et al. (2004) et John et al. (2002).

Genre Johannesbaptistia De Toni fil.

Johannesbaptistia pellucida (Dickie) Taylor et Drouet (Figure 12)

Syn. : Hormospora pellucida Dickie

Thalle linéaire cylindrique entouré d'un mucilage hyalin et formé de cellules rectangulaires à discoïdes. Les cellules, parfois finement granulées, sont arrangées sur une seule série. Leur diamètre est de 7 à $8 \mu \mathrm{m}$, leur longueur ne dépasse pas $4 \mu \mathrm{m}$. Le diamètre de la gaine mucilagineuse est de 3 à $4 \mu \mathrm{m}$. L'espèce présente les mêmes caractères que celle décrite par Da Rodda et Parodi (2005).

\section{Famille des Microcystaceae}

Genre Microcystis Lemmermann

Microcystis sp (Figure 13)

Colonie de très grande taille, formée de cellules sphériques pourvues de granulations; les cellules ne sont pas densément regroupées. Leur diamètre varie entre 2 et $3,5 \mu \mathrm{m}$.

\section{Famille des Borziaceae}

Genre Komvophoron Anagnostidis \& Komarek

Komvophoron sp (Figure 36)

Le trichome est droit, de couleur verte et à cellules cylindriques (ou en tonnelet) apparemment non jointives. Les cellules sont légèrement rétrécies vers leur milieu. Leur diamètre est de 1,5 $\mu \mathrm{m}$, leur longueur de $2 \mu \mathrm{m}$.

\section{Famille des Oscillatoriaceae}

Genre Lyngbya C. Agardh

Lyngbya confervoides C. Agardh (Figure 16)

De couleur verte, l'espèce présente des granulations et une gaine; le trichome n'est pas rétréci aux cloisons; ces cloisons apparaissent parfois comme des arcs. Les cellules sont rectangulaires avec un diamètre $11 \mu \mathrm{m}$ et une longueur d'environ $2 \mu \mathrm{m}$. Le diamètre du filament est de $13 \mu \mathrm{m}$. Les caractères observés chez cette espèce ont été également décrits par Crispino et Sant'Anna (2006), Da Rodda et Parodi (2005) et D'hont et Coppejans (1988).

Lyngbya semiplena J. Agardh ex Gomont (Figure 17)

Le filament présente une gaine ferme accolée au trichome. Ce dernier est formé de cellules rectangulaires avec beaucoup de granulations noirâtres. Les granulations sont plus nombreuses au niveau des cloisons. Le trichome est très légèrement constricté aux cloisons. Le diamètre des cellules est de $9 \mu \mathrm{m}$, leur longueur de 2-3 $\mu \mathrm{m}$. La description de l'espèce correspond à celle de Crispino et Sant'Anna (2006) et de Branco et al. (2003).

Lyngbya sp 1 (Figure 18)

L'espèce présente un filament droit avec un trichome constricté au niveau des cloisons. Le filament, à gaine ferme ou collée au trichome, a un diamètre de $5 \mu \mathrm{m}$. Les cellules sont rectangulaires avec environ 1,5 $\mu \mathrm{m}$ de long.

Lyngbya sp 2 (Figure 19)

L'espèce présente un trichome droit entouré d'une gaine de $1 \mu \mathrm{m}$ de diamètre. Le trichome, de couleur vert-jaune, est dépourvu de granules au niveau des cloisons. Les cellules sont rectangulaires avec 3-4 $\mu \mathrm{m}$ de diamètre et $1 \mu \mathrm{m}$ de longueur.

Genre Oscillatoria Vaucher

Oscillatoria acuta Bruhl et Biswas ex Geitler (Figure 20)

Le trichome est de couleur verte et est caractérisé par un apex effilé (cellule apicale conique) et incurvé. Les cellules granulées 
sont carrées à légèrement cylindriques avec 4$5 \mu \mathrm{m}$ de diamètre et 4-5 $\mu \mathrm{m}$ de longueur. La description est identique à celle d'Alvarez et al. (2000) et Compère (1974). Cependant les cellules sont carrées chez Compère (1974) mais moins longues que larges chez Alvarez et al. (2000).

Oscillatoria articulata Gardner (Figure 21)

Syn. : Phormidium articulatum (Gard.) Anag. \& Kom.

Le trichome est droit, avec des constrictions très légères aux articulations; les cellules sont plus ou moins carrées. Leur diamètre est de $3 \mu \mathrm{m}$ alors que leur longueur varie entre 2 et $2,5 \mu \mathrm{m}$; notre échantillon a le même ordre de grandeur que celui de Karol (1995) et Compère (1974).

Oscillatoria chlorina Kützing ex Gomont (Figure 22)

Trichome droit de couleur verte à «bleu-verdâtre » avec des granulations; les cloisons sont nettes et les constrictions ne sont pas très accentuées. Les cellules sont carrées à cylindriques; le diamètre est de $5 \mu \mathrm{m}$ et leur longueur de 5-6 $\mu \mathrm{m}$. Nos échantillons sont identiques à ceux de Naz et al. (2004), de Couté (1979) et de Compère (1974).

Oscillatoria limosa C. Agardh ex Gomont (Figure 23)

Le trichome ne présente pas de rétrécissement au niveau des articulations. Il est de couleur brunâtre ; la cellule apicale est hémisphérique et les autres cellules rectangulaires; elles sont toutes granulées. Leur diamètre est de $17 \mu \mathrm{m}$ avec des longueurs de 2-3 $\mu \mathrm{m}$. Ces caractères morphologiques ont été retrouvés dans la description des espèces rencontrées par Da Rodda et Parodi (2005) et D'hont et Coppejans (1988).

Oscillatoria subbrevis Schmidle (Figure 24)

Le trichome est droit de couleur vertnoirâtre avec beaucoup de granulations; les cellules sont rectangulaires avec des cloisons nettes. Leur diamètre est de $5 \mu \mathrm{m}$ et leur longueur de $3 \mu \mathrm{m}$. Nos échantillons ont le même diamètre que ceux de John et al. (2002) mais les cellules de ces derniers ont une longueur de 1-2 $\mu \mathrm{m}$.

Oscillatoria nigroviridis Thwaites ex Gomont. (Figure 25)
Le trichome est de couleur verte avec de nombreuses granulations ; les cellules sont rectangulaires mais la cellule apicale est hémisphérique. On note une constriction au niveau des cloisons. Le diamètre des cellules est de 5-6 $\mu \mathrm{m}$, avec une longueur de 1,5-2,5 $\mu \mathrm{m}$. Ces caractères ont été décrits par Da Rodda et Parodi (2005) et D'hont et Coppejans (1988).

Oscillatoria sp (Figure 26)

Le trichome de l'espèce est droit et très légèrement constricté aux cloisons; les cellules sont plus ou moins rectangulaires. Leur diamètre est de $3 \mu \mathrm{m}$ et leur longueur de $2 \mu \mathrm{m}$.

Genre Blennothrix Kützing ex Anagnostidis \& Komárek

Blennothrix lyngbyacea (Gomont) Anagn. \& Komàrek (Figure 27)

Syn.: Hydrocoleum lyngbyaceum Kütz. ex Gomont

L'espèce est formée d'un trichome non constricté de couleur verte, entourée d'une gaine et s'atténuant vers l'apex. Il a un diamètre de 13-14 $\mu \mathrm{m}$; les cellules granulées sont rectangulaires avec une longueur de 1,5-2 $\mu \mathrm{m}$. La cellule apicale est couverte d'un calyptre lentiliforme aplati (diamètre $=6 \mu \mathrm{m}$ ). La gaine est jaune-clair et est épaisse de 5 à 7 $\mu \mathrm{m}$. L'espèce a les mêmes ordres de grandeur et les mêmes caractères morphologiques que celle de Crispino et Sant'Anna (2006).

\section{Famille des Phormidiaceae}

Genre Phormidium Kütz. Ex Gom.

Phormidium autumnale (Agardh) Trevisan ex Gomont 1892 (Figure 28)

Le trichome, de couleur verte, est légèrement incurvé; il n'est pas rétréci au niveau des cloisons et présente des granulations. Chaque cellule est divisée par une cloison incomplète. Les cellules sont rectangulaires, de diamètre $5 \mu \mathrm{m}$ et de longueur égale à $3 \mu \mathrm{m}$. Les caractères observés ont été décrits par Matula et al. (2007).

Phormidium corium (Agardh) Gomont (Figure 29)

Syn. : Lyngbya corium (Gom.)

Syn. : Oscillatoria corium Agardh 
L'espèce présente un trichome de couleur bleu-vert ou vert sombre, non rétréci et entouré d'une sorte de gaine. Les cellules sont carrées à cylindriques et présentent ici des granulations. Elles ont $5 \mu \mathrm{m}$ de diamètre et 5 à $7 \mu \mathrm{m}$ de long. Notre échantillon présente des caractères communs avec ceux de Crispino et Sant'Anna (2006), de Da Rodda et Parodi (2005) et de Mirande et Tracanna (2004).

Phormidium hamellii (Frémy) Anag. \& Kom. (Figure 30)

Syn. : Oscillatoria hamellii Frémy

Le trichome est droit, court, de couleur verte et pourvu de granulations. Il est très légèrement rétréci au niveau des articulations. Les cellules ou articles sont cylindriques avec $5 \mu \mathrm{m}$ de diamètre. Leur longueur est de 6-7 $\mu \mathrm{m}$. Cette espèce présente des caractères communs avec celles décrites par Tell (1994). Couté (1979) et Compère (1974) l'ont dénommée Oscillatoria hamellii Frémy.

Phormidium retzii Ag. (Figure 31)

Syn. : Oscillatoria retzii Agardh

L'espèce présente un trichome droit pourvu de granulations et de pseudovacuoles. Le trichome est légèrement rétréci aux articulations et présente un apex arrondi. Les cellules sont plus ou moins carrées. Leur diamètre est de 5-6 $\mu \mathrm{m}$, leur longueur de 4 à 5 $\mu \mathrm{m}$. L'espèce est identique à celles décrites par Hussain et al. (2009) et Da Rodda et Parodi (2005).

Genre Planktothrix Anagnostidis et Komárek Planktothrix isothrix Komárek et Komárková (Skuja) comb. Nova (Figure 32)

Syn. : Planktothrix mougeotii (Bory ex Gom.) Anagn. \& Kom.

Syn.: Oscillatoria mougeotii Kützing ex Lemmermann

Syn.: Oscillatoria agardhii var. isothrix Skuja

Espèce à trichome droit, sans constriction et à apex non atténué. Les cellules sont rectangulaires et pourvues de très nombreuses vacuoles à gaz. Le diamètre du trichome est de $9 \mu \mathrm{m}$, la longueur des cellules de 5-6 $\mu \mathrm{m}$. Ces caractères morphologiques ont été retrouvés chez l'espèce décrite par Komárek et Komárková (2004).
La description est également identique à celle de Compère (1974) et à celle de John et al. (2002) qui l'ont nommé respectivement Oscillatoria mougeotii et Oscillatoria agardhii var. isothrix.

Planktothrix raciborskii (Wołosz.) Anagn. \& Kom. (Figure 33)

Syn. : Planktothricoides raciborskii (Wołosz.) Suda \& Watanabe

Syn. : Oscillatoria raciborskii Wołosz

Le trichome, de couleur verte, n'est pas rétréci au niveau des cloisons et présente des aérotopes ou pseudovacuoles. Les cellules sont rectangulaires, de diamètre 4-4,5 $\mu \mathrm{m}$ et de longueur égale à 2-3 $\mu \mathrm{m}$. Les cellules terminales, coniques, ont $3 \mu \mathrm{m}$ de diamètre et 4-5 $\mu \mathrm{m}$ de longueur. La description est identique à celle de Komárek et Komárková (2004).

\section{Famille des Pseudanabaenaceae}

Genre Leptolyngbya Anagnostidis et Komárek Leptolyngbya cf. foveolarum Anagn. et Kom. (Figure 34)

Syn. : Lyngbya foveolarum (Gom.) Hansg.

Syn. : Phormidium foveolarum Gomont

Le filament est de 1,5-2 $\mu \mathrm{m}$ de diamètre. Le trichome, de couleur verte, est à peine rétréci aux articulations. Les cellules sont d'environ $1 \mu \mathrm{m}$ de long. Notre échantillon a le même ordre de grandeur que celui de Matula et al. (2007).

Genre Pseudanabaena Lauterborn

Pseudanabaena catenata Lauterb. (Figure 35)

Le trichome est formé de cellules en tonnelet apparemment non jointives; les cellules ont $1,5 \mu \mathrm{m}$ de diamètre et 3-3,5 $\mu \mathrm{m}$ de longueur.

Pseudanabaena sp

L'espèce présente un trichome droit de couleur verdâtre et formé de cellules cylindriques ; la cellule terminale est arrondie. Les rebords du trichome sont plus verts que le centre qui est parfois clair; Le diamètre des cellules est de $2 \mu \mathrm{m}$, leur longueur de 3-3,5 $\mu \mathrm{m}$.

Pseudanabaena spl (Figure 15). 
Qิ宀
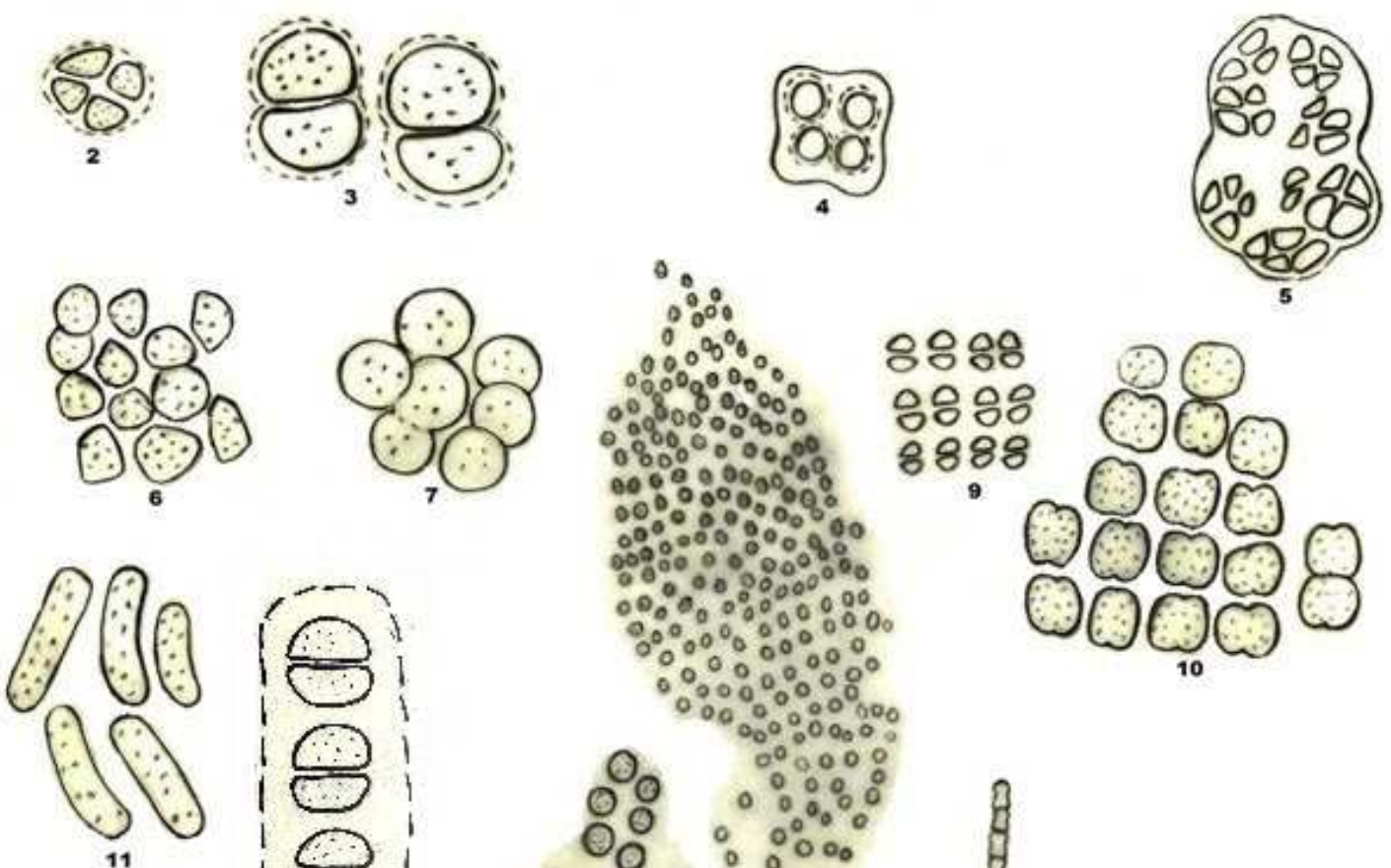

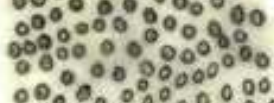
0000.0000000 .0000000000

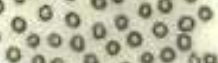
88888
8888
8888 $00: 000 \%$

mC
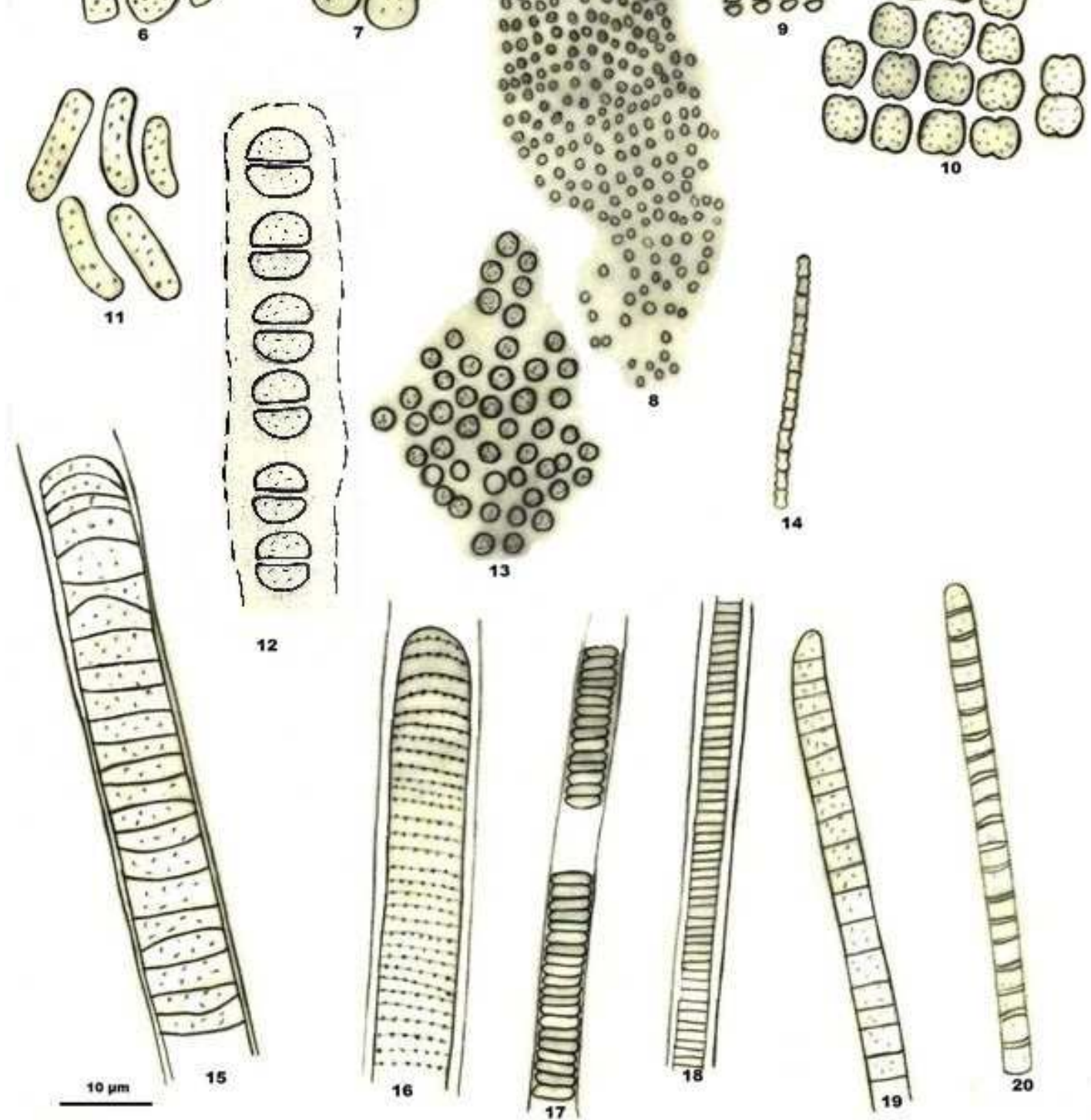

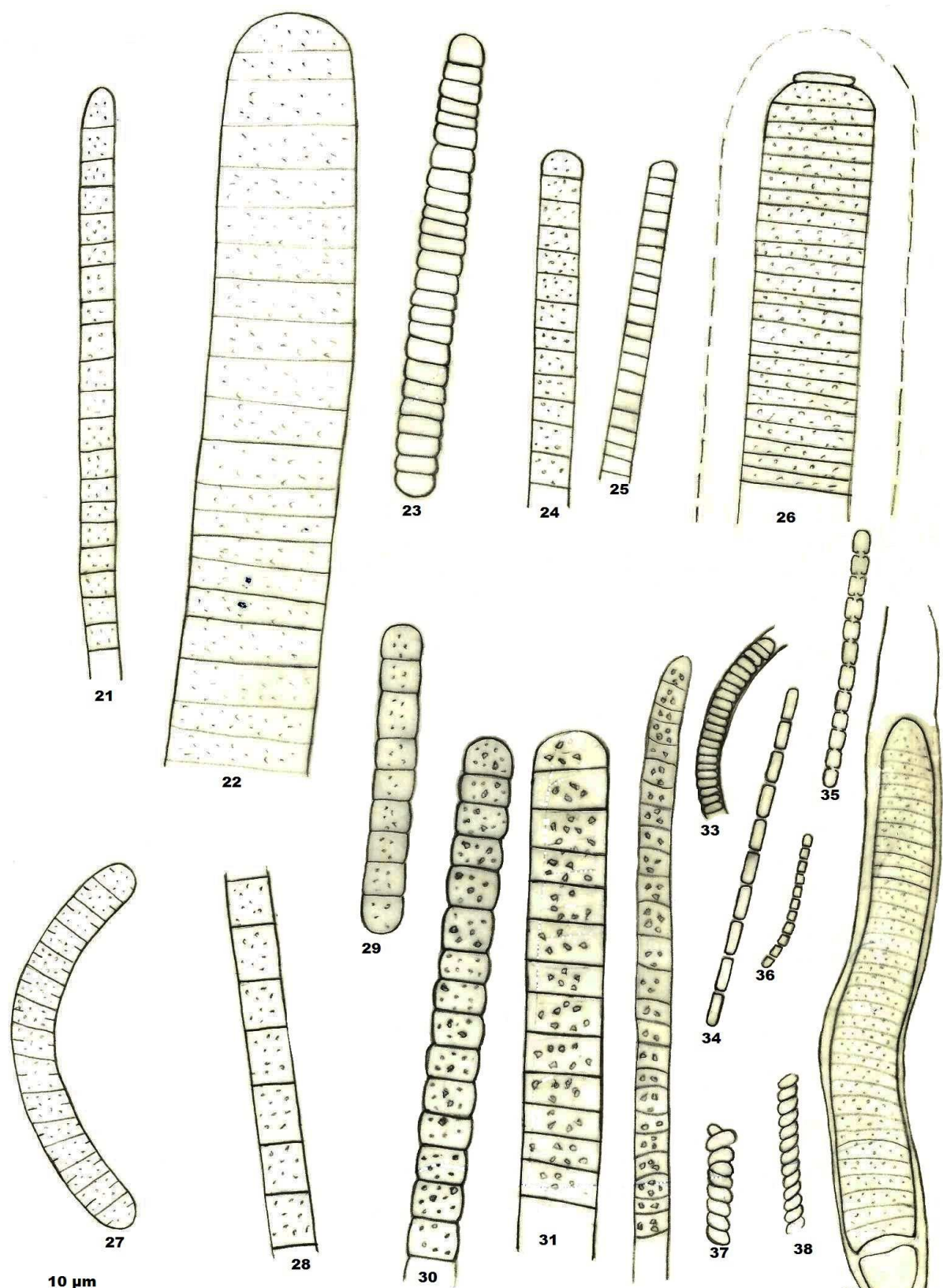

$10 \mu \mathrm{m}$
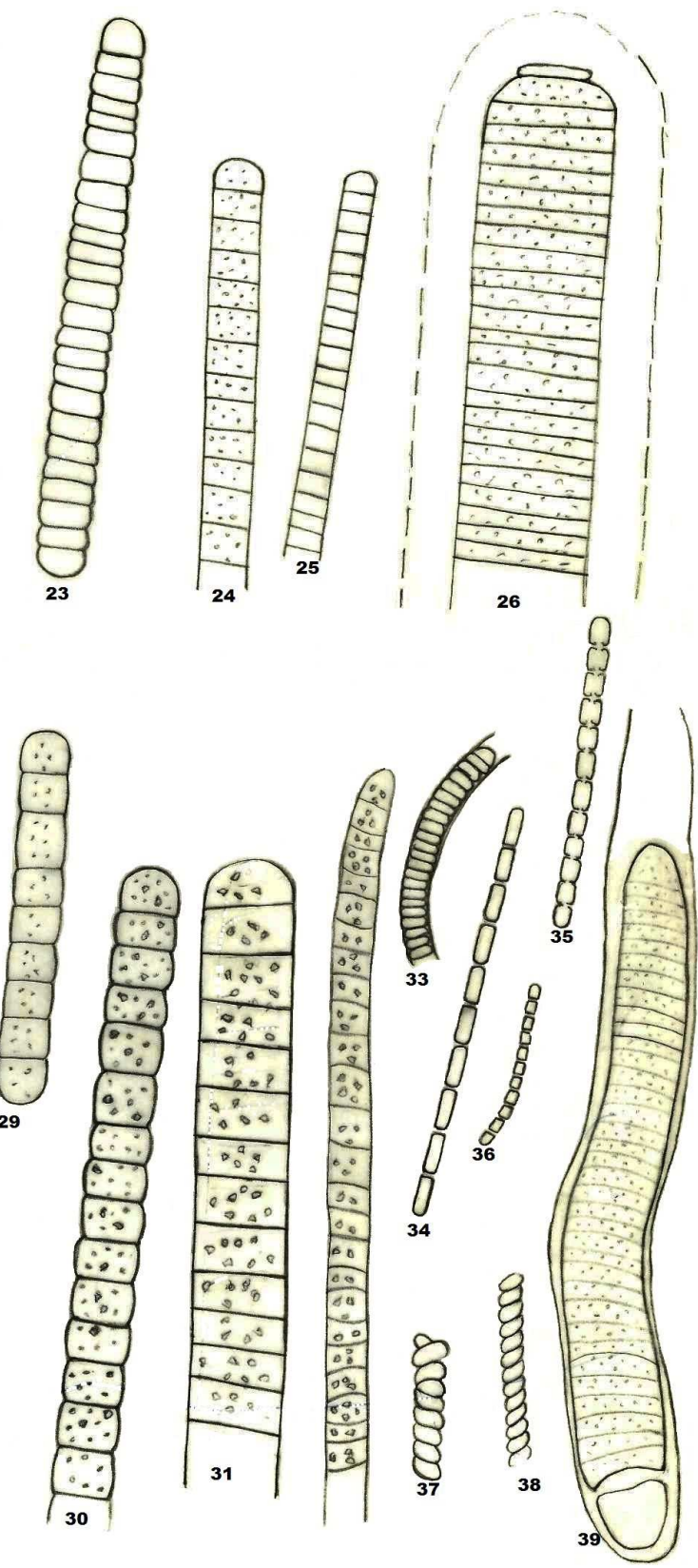

Figure 2: Chroococcus minor

Figure 3 : Chroococcus minutus, Figure 4 Chroococcus varius, Figure 5 : Myxosarcina sp Figure 6 : Pleurocapsa sp, Figure 7 : Aphanocapsa littoralis, Figure $8:$ Aphanocapsa marina Figure 9: Merismopedia minima, Figure 10: Merismopedia mediterranea, Figure 11: Rhabdoderma lineare; Figure 12: Johannesbaptistia pellucida, Figure13: Microcystis sp, Figure 14 : Komvophoron $s p$; Figure 15 : Lyngbya confervoides, Figure 16 : Lyngbya semiplena, Figure 17 : Lyngbya sp 1

Figure 18 : Lyngbya sp2, Figure 19 ; Oscillatoria acuta, Figure 20 : Oscillatoria articulata

Figure 21 : Oscillatoria chlorina, Figure 22 : Oscillatoria limosa, Figure 23 : Oscillatoria subbrevis 
Figure 24 : Oscillatoria nigroviridis, Figure 25 : Oscillatoria sp, Figure 26 : Blennothrix lyngbyacea Figure 27: Phormidium autumnale, Figure 28: Phormidium corium, Figure 29: Phormidium hamellii; Figure 30 : Phormidium retzii, Figure 31 : Planktothrix isothrix, Figure32 : Planktothrix raciborskii, Figure 33: Leptolyngbya cf foveolarum, Figure 34: Pseudanabaena catenata, Figure35: Pseudanabaena sp ; Figure 36: Pseudanabaena sp 1, Figure37: Spirulina subsalsa, Figure 38 : Spirulina labyrinthiformis ; Figure39 : Calothrix sp

Le trichome est formé de cellules carrées à légèrement cylindriques, à cotés arrondis (en tonnelet). Les dimensions des cellules sont $1 \mu \mathrm{m} \times 1-1,5 \mu \mathrm{m}$.

Genre Spirulina Turpin ex Gomont

Spirulina subsalsa Oersted. ex Gomont (Figure 37)

Le trichome de l'espèce est hélicoïdal avec un contenu cellulaire vert-jaune; le diamètre du trichome est d'environ $1 \mu \mathrm{m}$ et celui des spires contigües est de $3 \mu \mathrm{m}$. Notre échantillon est identique à ceux de Da Rodda et Parodi (2005) et D'hont et Coppejans (1988).

Spirulina labyrinthiformis Kütz. ex Gomont (Figure 38)

Le trichome de l'espèce est hélicoïdal avec un contenu cellulaire vert-jaune. Le diamètre du trichome est légèrement inférieur à $1 \mu \mathrm{m}$. Le diamètre des spires de 1,5-2 $\mu \mathrm{m}$. L'espèce est décrite de la même façon que Crispino et Sant'Anna (2006).

\section{Famille des Rivulariaceae}

Genre Calothrix Agardh ex Bornet et Flahault Calothrix sp (Figure 39)

Le filament est solitaire, pourvu d'une gaine et effilé au sommet; il présente un hétérocyste basal. Le trichome est de couleur brun-rouge et présente des granulations. Les cellules sont rectangulaires ; le diamètre à la base est de 11-12 $\mu \mathrm{m}$; la longueur des cellules varie entre 2 et $3 \mu \mathrm{m}$. L'hétérocyste basal à un diamètre de 6-7 $\mu \mathrm{m}$ et une longueur d'environ $6 \mu \mathrm{m}$.

\section{DISCUSSION}

Il ressort des résultats obtenus que la communauté de cyanophytes est représentée par 38 taxa. Sur les 28 taxa identifiés jusqu'au niveau espèce, 17 ont été décrits pour la $1^{\text {ere }}$ fois au Sénégal par comparaison aux travaux de De Poucques (1956), de Dia et Reynaud (1982), de Compère (1991), de Cogels et Gac (1995), de Carlbro (1999), de Berger et al. (2005), de Ba et al. (2006) et de Gueye et al. (2013). Ce sont: Chroococcus varius, Aphanocapsa marina, Aphanocapsa littoralis, Merismopedia minima, Merismopedia mediterranea, Johannesbaptistia pellucida, Lyngbya confervoides, Lyngbya semiplena, Oscillatoria limosa, Oscillatoria nigroviridis, Blennothrix lyngbyacea, Phormidium autumnale, Phormidium corium, Phormidium retzii, Planktothrix isothrix, Planktothrix raciborskii, et Spirulina labyrinthiformis.

Par comparaison aux travaux antérieurs sur la biodiversité des microphytes des eaux douces du Sénégal, il a été noté la présence en eau douce et salée de certaines espèces de Cyanophyceae. Il s'agit de Chroococcus minor et Chroococcus minutus citées par Gueye et al. (2013), Rhabdoderma lineare par Ba (2006), Oscillatoria chlorina par Berger et al. (2005), Pseudanabaena catenata par Berger et al. (2005), Ba et al. (2006) et Gueye et al. (2013), Leptolyngbya cf. foveolarum par Compère (1991) sous le nom Lyngbya foveolarum, Oscillatoria acuta, Oscillatoria articulata et Phormidium hamellii par Compère (1991), Spirulina subsalsa par Reynaud et Roger (1981) et Compère (1991), 
et Oscillatoria subbrevis par Compère (1991) et Berger et al. (2005).

En effet, les Cyanophyta forment le groupe supportant les plus grandes amplitudes de salinité. Elles comprennent une proportion importante d'espèces très euryhalines trouvées aussi bien dans les eaux douces que dans les milieux eu- ou hypercarbonatés (Iltis, 1973).

En plus la diversité spécifique des Cyanophyta présents au niveau de cet écosystème a montré une plus grande représentativité des formes non hétérocystées. En effet seul le genre Calothrix avec l'espèce Calothrix sp a été recensé. Ceci peut s'expliquer par l'abondance de l'azote qui proviendrait des quantités d'eau usées produites par les ménages et qui y sont directement déversées. Ces résultats sont en conformité avec les travaux de Schindler et son équipe en 1977 qui ont démontré en conditions expérimentales que les cyanophytes fixateurs d'azote augmentaient considérablement lorsque le ratio NT/PT était abaissé. De même, la théorie de Blomqvist et al. (1994) suggère que le succès des cyanophytes non-fixateurs d'azote est influencé par la présence de la forme inorganique d'azote $\left(\mathrm{NO}^{3-}\right)$.

\section{REMERCIEMENTS}

Cette étude a été réalisée avec le soutien de la Fondation Mitsubishi.

\section{REFERENCES}

Abbott IA, Hollenberg GJ. 1992. Marine Algae of California. Stanford University Press: California.

Alvarez SB, Bazan GI, Wenzel MT. 2000. Hormogonales (Cyanophyta) from El Guanaco pond (La Pampa, Argentina). Darwiniana, 38(3-4): 279-284.

Amossé A. 1970. Diatomées marines et saumâtres du Sénégal et de la Côte
d'Ivoire. Bulletin IFAN, sér. A, 32(2) : 289-311.

Ba N. 2006. La communauté phytoplanctonique du Lac de Guiers (Sénégal): types d'associations fonctionnelles et approches expérimentales des facteurs de régulation. Thèse de Doctorat de $3^{\mathrm{e}}$ Cycle, Université Cheikh Anta Diop, Dakar, p. 144.

Ba AT, Noba K. 2001. Flore et Biodiversité végétale au Sénégal. Sécheresse, 12(3) : 149-155.

Ba N, Noba K, Dieye M, Kane A, Mbaye MS, Sarr RS, Kane M, Hane PD, BA AT. 2006. Etude systématique des Cyanophycées de quelques rizières de la moyenne vallée et du delta du fleuve Sénégal. Webbia, 61(2) : 261-269.

Berard-Therriault L, Poulin M, Bosse L. 1999. Guide d'Identification du Phytoplancton Marin de l'Estuaire et du Golfe du SaintLaurent: Incluant Egalement certains Protozoaires. NRC Research Press: Ottawa.

Berger C, Couté A, Ba N, Gugger M. 2005. Cyanobacterial taxa of the Senegal River hydrosystem (northern Senegal, West Africa). Algol. Stud., 117: 147-176.

Bodard M, Mollion J. 1974. La végétation infralittorale de la petite côte sénégalaise. Bull. Soc. Phycol. France, 19 : 193 - 221.

Bodian MY. 2000. Systématique et biologie des algues macrophytes collectées pendant la période hivernale dans la zone incluant Dakar et la Petite Côte (Août et décembre 1998). Mémoire de D.E.A, Université Cheikh Anta Diop, Dakar, p. 177.

Blomqvistl P, Pettersson A, Hyenstrand P. 1994. Ammonium-nitrogen: A key regulatory factor causing dominance of non-nitrogen-fixing cyanobacteria in aquatic systems. Archiv fur Hydrobiologie, 132(2): 141-164. 
Branco LHZ, Moura AN, Silva AC, Bittencourt-Oliveira MC. 2003. Biodiversidade e considerações biogeográficas da cyanobacteria de uma área de Manguezal do estado de Pernambuco, Brasil. Acta Bot. Bras., 17(4): 585-596.

Carlbro International. 1999. Etude bathymétrique et limnologique du lac de Guiers. Rapport de synthèse. Hydroconsult international, SGPRE. 119.

Cogels FX, Gac JY. 1995. Lake of Guiers (Senegal, West Africa). ILEC Newsletter, 20: 6-7.

Compère P. 1991. Contribution à l'étude des algues du Sénégal. Algues du lac de Guiers et du Bas Sénégal. Bulletin $d u$ Jardin Botanique du Belgique, 61: 171267.

Crispino LMB, Sant'Anna CL. 2006. Cianobactérias marinhas bentônicas de ilhas costeiras do Estado de São Paulo. Brasil Revista Brasil. Bot., 29(4) : 639656.

D’hont D, Coppejans E. 1988. Cyanophycées des côtes du nord de la France et de la Belgique. Bull. Soc. Roy. Bot. Belg., 121: 35-54.

Da Rodda C, Parodi ER. 2005. Cyanophyceae epipèlicas de la Marisma «El Cangrejal » en el estuario de Bahìa Blanca (Buenos Aires, Argentina). Bol. Soc. Argent. Bot., 40(3-4) : 157-168.

Dangeard P. 1952. Algues de la Presqu'île du Cap Vert (Dakar) et ses environs. Botaniste, 36: 193-329.

De Poucques ML. 1956. Examen de la flore algale de quelques mares du Sénégal. Bull. Soc. Sci. Nancy., 15(2) :77-83.

Dia A, Reynaud PA. 1982. Le phytoplancton du lac de Guiers : approche qualitative et quantitative. Cahier ORSTOM, Série Biologie, 45: 35-47.
Dia A. 1983. Observations océanographiques effectuées en 1982. Centre de Recherches Océanographiques de Dakar-Thiaroye. Archive Scientifique du Crodt., 126 : 77.

Diedhiou I. 2010. Contribution à la connaissance de la biodiversité des algues macrophytes marines du Sénégal, cas des îles de Dakar: Gorée, Madeleines, Ngor et Yoff Tonghor. Mémoire de Master. Université Cheikh Anta Diop, Dakar, p. 34.

Guermeur P 1954. Diatomées de l'AOF (Première liste: Sénégal), IFAN Catalogue, 12 : 137.

Gueye M, Ba N, Noba K, Mbaye MS., Hane PD., Bassene C, Kane M, Kane A. 2013. Cyanophytes et microalgues (Diatomées exclues) du Parc National des Oiseaux du Djoudj (Sénégal). Scripta Botanica Belgica, 50 : 272-286.

Harper JT, Garbary DJ. 1997. Marine Algae of Northern Senegal: the flora and its bibliography. Botanica Marina, 40: 129138.

Hussain F, Masud, Shah SM, Fazli-e-Hadi, Zaman A, Wazir SM. 2009. Some blue green algae from rice fields of Asota Sharif District Swabi, Pakistan. Pak. J. Pl. Sci., 15 (1): 45-57.

John DM, Whitton BA, Brook AJ. 2002. The Freshwater Algal Flora of the British Isles: an Identification Guide to Freshwater and Terrestrial Algae. Cambridge University Press: London; 1.

Komárek J et Hauer T. 2011. CyanoDB.cz On-line database of cyanobacterial genera. - Word-wide electronic publication, Univ. of South Bohemia \& Inst. of Botany AS CR, http://www.cyanodb.cz. Consulté le 12 janvier 2012.

Komárek J, Komárková J. 2004. Taxonomic review of the cyanoprokaryotic genera 
Planktothrix and Planktothricoides. Czech Phycology, Olomouc., 4: 18.

Leuduger-Fortmorel G. 1898. Diatomées marines de la Côte Occidentale d'Afrique. Mémoires de la Société d'Emulation des Côtes-du-Nord. Imprimerie Francisque Guyon St., Brieuc. Matula J, Pietryka M, Richter D et Wojtuń B. 2007. Cyanoprokaryota and algae of Arctic terrestrial ecosystem in the Hornsund area, Spitsbergen. Pol. Polar. Res., 28(4) : 283-315.

Mepn. 2007. Rapport national sur l'état de l'environnement marin et côtier. 70.

Naskar N, Naskar KR, Sen CR. 2008. Brackish water Oscillatoriaceae fron North 24-Parganas, West Bengal, India. Bangladesh J. Plant Taxon., 15(1) : 3138.

Naz S, Masud-ul-hasan, Shameel M. 2004. Taxonomic study of Chroocophyceae (Cyanophyta) from Northern areas of Pakistan. Pak. J. Bot., 36(2): 247-281.

Nogueira NMC, Ferreira-Correia MM. 2001. Cyanophyceae/Cyanobacteria in red mangrove forest at Mosquitos and Coqueiros estuaries, Sao Luis, State of Maranhao, Brazil. Braz. J. Biol., 61(3): 347-356.
Park M, Dixon PT. 1964. A revised check-list of British marine algae. J. Mar. Biol. Ass. U.K., 44: 499- 542.

Reynaud PA, Roger PA. 1981. Variations saisonnières de la flore algale et de l'activité fixatrice d'azote dans un sol engorgé de bas de dune. Rev. Ecol. Biol. Sol., 18(1) : 9-27.

Sant'Anna CL, Azevedo MTP, Senna PAC, Komárek J, Komárková J. 2004. Planktic Cyanobacteria from São Paulo State, Brazil: Chroococcales. Revista Brasil. Bot., 27(2): 213-227.

Sant'Anna CL, Melcher SS, Carvalho MC, Gemelgo MP, Azevedo MTP. 2007. Planktic Cyanobacteria from upper Tietê basin reservoirs, SP, Brazil. Revista Brasil. Bot., 30(1): 1-15.

Schindler D. W. 1977. Evolution of phosphorus limitation in lakes. Sciences, 195: 260-262.

Silva PC, Basson PW, Moe RL. 1996. Catalogue of the Benthic Marine Algae of the Indian Ocean. University of California Press: California.

Tell G. 1994. Planktonic Cyanophyceae from northeastern Argentina. Rev. Hydrobiol. trop., 27(4): 347-360. 